\title{
CAMPOS IMPREGNADOS COM IODO EM CIRURGIA DA COLUNA: IMPACTO NA INFECÇÃO DE SÍTIO CIRÚRGICO
}

\author{
Iodine-impregnated incision drapes in spine surgery: impact on surgical site infection \\ Paños quirúrgicos impregnados com yodo em cirugía de coluna: \\ impacto en la infección del sitio quirúrgico
}

\author{
Eliane Carlosso Krummenauer ${ }^{1 *}$ (D), Jane Dagmar Pollo Renner ${ }^{2}$ (D), Rochele Mosmann Menezes ${ }^{3}$ (D), \\ Telmo Tiburcio Fortes Lima ${ }^{4} \mathbb{D}$, Marcelo Carneiro ${ }^{5}$ (B)
}

RESUMO: Objetivo: Avaliar o impacto de campos adesivos impregnados com iodo (CAII) na prevenção de infecção de sítio cirúrgico (ISC) de coluna vertebral. Método: Coorte retrospectiva que comparou desfecho de ISC em pacientes que utilizaram CAII com os que não usaram, de 2015 a 2019 . Resultados: A frequência geral de ISC foi de $16,7 \%$, com a taxa de ISC para os que utilizaram CAII de $40 \%$ e, entre os que não usaram, de $60 \%$; $p=0,728$; intervalo de confiança de 95\% (IC95\%) 0,19-3,11. A normotermia foi o único fator protetor independente para ISC ( $\mathrm{p}=0,043)$. O tratamento de complicações infecciosas acarretou o incremento de custo hospitalar de $83,6 \%$ a cada dia de atendimento. Os pacientes que utilizaram CAII tiveram 10 ( $\pm 4,9$ ) dias a menos de permanência hospitalar. Conclusões: Os resultados sugerem que o uso de CAII não foi associado a menor risco de ISC. Esses dados podem ser úteis para o planejamento cirúrgico e a segurança do paciente.

Palavras-chave: Campos cirúrgicos. Coluna vertebral. Infecção da ferida cirúrgica. Controle de infecções. Produtos com ação antimicrobiana.

ABSTRACT: Objective: To evaluate the impact of iodine-impregnated incision drapes (IIIDs) to prevent surgical site infection (SSI) in the spine. Method: Retrospective cohort study comparing SSI outcome in patients in which IIIDs were and were not used, from 2015 to 2019 . Results: The overall frequency of SSI was $16.7 \%$, with SSI rate among patients using and not using IIIDs of $40 \%$ and $60 \%$, respectively; $\mathrm{p}=0.728$; $95 \%$ confidence interval (95\%CI) 0.19 3.11. Normothermia was the only independent protective factor for SSI $(\mathrm{p}=0.043)$. The treatment of infectious complications resulted in hospital costs increase of $83.6 \%$ each day of care. Patients who were treated with IIIDs stayed 10 days less $( \pm 4.9)$ in hospital. Conclusions: The results suggest that the use of IIIDs was not associated with a lower risk of SSI. These data can be useful for surgical planning and patient safety.

Keywords: Surgical drapes. Spine. Surgical wound infection. Infection control. Products with antimicrobial action.

RESUMEN: Objetivo: Evaluar el impacto de las paños quirúrgicos adhesivos impregnados de yodo (IIIDS) en la prevención de la infección del sitio quirúrgico (ISQ) de la columna. Método: Cohorte retrospectiva que comparó el resultado de ISQ en pacientes que usaron IIIDS con los que no lo hicieron, de 2015 a 2019. Resultados: La frecuencia general de ISQ fue del 16.7\%, con una tasa de ISQ para los que usaron IIIDS del $40 \%$ y, entre los que no lo usaron, $60 \% ; \mathrm{p}=0,728$; Intervalo de confianza del 95\% (IC 95\%) 0,19-3,11. La normotermia fue el único factor protector independiente para la ISQ (p = 0,043). El tratamiento de las complicaciones infecciosas supuso un aumento de los costes hospitalarios del 83,6\% por día de atención. Los pacientes que utilizaron IIIDS tuvieron $10( \pm 4,9)$ días menos de estancia hospitalaria. Conclusiones: Los resultados sugieren que el uso de IIIDS no se asoció con un menor riesgo de ISQ. Estos datos pueden ser útiles para la planificación quirúrgica y la seguridad del paciente.

Palabras clave: Paños quirúrgicos. Columna vertebral. Infección de la herida quirúrgica. Control de infecciones. Productos con acción antimicrobiana.

'Mestre em Promoção da Saúde pela Universidade de Santa Cruz do Sul (Unisc). Enfermeira do Hospital Santa Cruz - Santa Cruz do Sul (RS), Brasil.

2Doutora em Biologia Celular e Molecular pela Pontifícia Universidade Católica do Rio Grande do Sul. Professora do Programa Stricto Sensu em Promoção da Saúde e dos Departamentos de Ciências da Saúde e Ciências da Vida da Unisc - Santa Cruz do Sul (RS), Brasil.

${ }^{3}$ Mestre em Promoção da Saúde pela Unisc. Membro da Comissão de Controle de Infecção e Epidemiologia Hospitalar do Hospital Santa Cruz - Santa Cruz do Sul (RS), Brasil.

"Médico Neurocirurgião do Hospital Santa Cruz - Santa Cruz do Sul (RS), Brasil.

${ }^{5}$ Pós-doutorando em Educação pela Unisc. Professor do Programa Stricto Sensu em Promoção da Saúde e dos Departamentos de Ciências da Saúde e Ciências da Vida da Unisc - Santa Cruz do Sul (RS), Brasil.

*Autora correspondente: elianekrummenauer@gmail.com

Recebido: 14/01/2021 - Aprovado: 01/09/2021

https://doi.org/10.5327/Z1414-4425202100030004 


\section{INTRODUÇÃO}

As cirurgias de coluna são procedimentos em crescimento em virtude do aumento da expectativa média de vida ${ }^{1}$. Quando indicadas, elas têm o objetivo de salvar ou melhorar a qualidade de vida, mas a falta de segurança em relação a alguns fatores não controlados pode causar eventos como infecções, incapacidades e morte ${ }^{2}$. Apesar da aplicação de protocolos para a prevenção de infecção de sítio cirúrgico (ISC), esta continua sendo causa significativa de morbidade pós-operatória, mortalidade e aumento de custos. Alguns procedimentos que envolvem próteses possuem maior risco de complicações, além de prolongarem a internação e aumentarem as reinternações para a execução de novos procedimentos e terapias ${ }^{3}$.

Diante desse cenário imposto aos pacientes, destaca-se a necessidade de esforços para criar estratégias de prevenção de ISC. É importante a identificação das condições de risco, que são fatores clínicos ou estados que predispõem ao desenvolvimento da ISC, a fim de protagonizar ajustes para garantir a segurança cirúrgica ${ }^{2}$. Um dos aspectos relevantes é a exposição da ferida operatória ao ambiente durante o tempo de realização do procedimento. Essa situação, agregada a outras condições predisponentes, pode ser determinante para a ISC ${ }^{3}$. A prevenção de ISC está fundamentada em causas como carga bacteriana, virulência do agente, risco de infecção e defesa imunológica do paciente ${ }^{2}$.

Entre os vários elementos identificados para a prevenção de ISC, um dos procedimentos mais importantes é o preparo da pele, e existem poucos antissépticos para atender a essa recomendação. Os objetivos da utilização desses agentes químicos são remover os microrganismos, proporcionar a morte química e inibir o crescimento de microrganismos por meio de várias técnicas e combinações, a fim de reduzir a microbiota da pele (temporária e residente) durante o período que a cirurgia pode durar ${ }^{4}$.

Diante desse contexto multifatorial, fica evidente a necessidade de atenção multiprofissional e de ações interdisciplinares, além do uso de tecnologias adicionais, para prevenir esse agravo. O uso de campo adesivo impregnado com iodo (CAII) é uma opção para reduzir o microbioma residente cutâneo que persiste após a aplicação de preparações antissépticas clássicas ${ }^{4-6}$.

\section{OBJETIVOS}

\section{Objetivo primário}

- Avaliar o impacto de CAII na prevenção de ISC de coluna vertebral.

\section{Objetivos secundários}

- Descrever as características clínicas e os fatores de risco dos pacientes submetidos a cirurgias de coluna vertebral;

- Avaliar o custo-efetividade do uso de CAII na incidência de ISC da coluna vertebral;

- Verificar se a utilização dos CAII interferiu no tempo de internação hospitalar;

- Determinar a frequência de reações adversas cutâneas associadas ao uso de CAII.

\section{MÉTODO}

Trata-se de um estudo de coorte retrospectiva, que comparou 60 pacientes submetidos a cirurgias de coluna vertebral em relação ao uso ou não de CAII, de janeiro de 2015 a dezembro de 2019, em um hospital de ensino do interior do Rio Grande do Sul (Brasil). A instituição sede da pesquisa é um hospital filantrópico, que possui sete salas de cirurgia, com média de 622 procedimentos anestésico-cirúrgicos por mês e 2,6 cirurgias de coluna por mês durante o período do estudo.

Os pacientes eleitos para a pesquisa foram identificados por meio de um relatório de cirurgias extraído do sistema de atendimento institucional, o qual possibilitou avaliar e classificar as variáveis, bem como a utilização ou não de CAII. A inclusão dos sujeitos no estudo ocorreu para todos os pacientes maiores de 18 anos, submetidos à cirurgia de coluna vertebral, de classificação limpa, realizada no período pela única equipe cirúrgica da especialidade. A exclusão do estudo ocorreu com pacientes submetidos à cirurgia de coluna vertebral de classificação infectada. Os sujeitos foram analisados pelos prontuários eletrônicos e físicos, com o preenchimento de formulário com as variáveis do estudo, e classificados de acordo com a utilização ou não de CAII e o desfecho de ISC (Figura 1).

Os pacientes submetidos às cirurgias de artrodese, fratura de coluna, luxação de coluna, hérnia discal e laminectomia foram avaliados durante a internação e em longo prazo (90 dias após a alta) pelo Serviço de Controle de Infecção. Os critérios utilizados foram os estabelecidos pela Agência Nacional de Vigilância Sanitária (ANVISA) ${ }^{7}$ e pelo protocolo institucional de vigilância de ISC no pós-alta. Foram consideradas ISC superficial, ISC profunda e ISC de órgão e espaço. Os registros foram efetuados em módulo de sistema informatizado específico de controle de infecções e considerados para a análise da ISC. 
A Tabela 1 apresenta as variáveis preditoras de risco de ISC do sistema nervoso central definidas de acordo com a ANVISA ${ }^{7,8}$, pela classificação da American Society of Anesthesiologists (ASA) ${ }^{9}$ e também pelo índice de risco para ISC (IRIC) do National Nosocomial Infections Surveillance (NNIS) do Centers for Disease Control and Prevention (CDC) ${ }^{10}$. As variáveis coletadas para a análise foram: idade, sexo, convênio de atendimento (privado e não privado [Sistema Único de Saúde]), urgência cirúrgica, internação pré-cirúrgica e internação pré-cirúrgica em unidade de terapia intensiva (UTI), ASA, índice de massa corpórea (IMC), local da cirurgia, duração da cirurgia, preparo da pele, uso de CAII, profilaxia antimicrobiana (30 a 60 min antes da incisão), profilaxia estendida (24 horas), trauma cirúrgico, presença de infecção pré e pós-operatória, normotermia (35,5 a $38,3^{\circ} \mathrm{C}$ ), drenos até 24 horas no pós-operatório, internação pós-operatória em UTI e óbito.

$\mathrm{Na}$ análise das variáveis, a localização foi categorizada como "cirurgia alta" para os procedimentos em nível da coluna cervical, independentemente de níveis ou abordagem, e como "cirurgia baixa" para os restantes.

O CAII (Ioban ${ }^{\circledR} 2$; 3M, St. Paul, MN) é um campo com adesivo acrílico hipoalergênico, impregnado de iodo e sensível à pressão, que promove uma superfície estéril e ajuda a prevenir a migração de microrganismos para o sítio cirúrgico ${ }^{4}$.
A participação da pesquisa aconteceu por apenas uma equipe cirúrgica, a qual não apresentava critérios de utilização do CAII definidos.

Os custos de medicamentos, materiais (incluindo órteses e próteses), exames, equipamentos e estrutura hospitalar (hotelaria) foram mensurados. Consideraram-se para essa verificação todos os custos com a internação, exceto a remuneração pelo atendimento médico. Esses dados foram fornecidos pela instituição e reportam o referido valor do período do atendimento.

Os dados foram analisados no programa Statistical Package for the Social Sciences (SPSS IBM, Armonk, EUA), versão 23.0. Medidas de efeito foram utilizadas, como diferença de médias ou diferença de proporções, com seus respectivos intervalos de confiança de 95\% (IC95\%). A regressão linear múltipla foi aplicada para as variáveis que apresentaram $p<0,20$ na regressão linear simples, a fim de considerar fatores de risco para o desfecho. Os valores de $\mathrm{p}<0,05$ foram considerados significantes.

O projeto foi submetido, via plataforma Brasil, a apreciação no Comitê de Ética em Pesquisa (CEP) e foi aprovado sob parecer número 3.629.429, em 8 de outubro de 2019, atendendo às Diretrizes e Normas Regulamentadoras de Pesquisas Envolvendo Seres Humanos (Resolução 466/ 2012 do Conselho Nacional de Saúde).

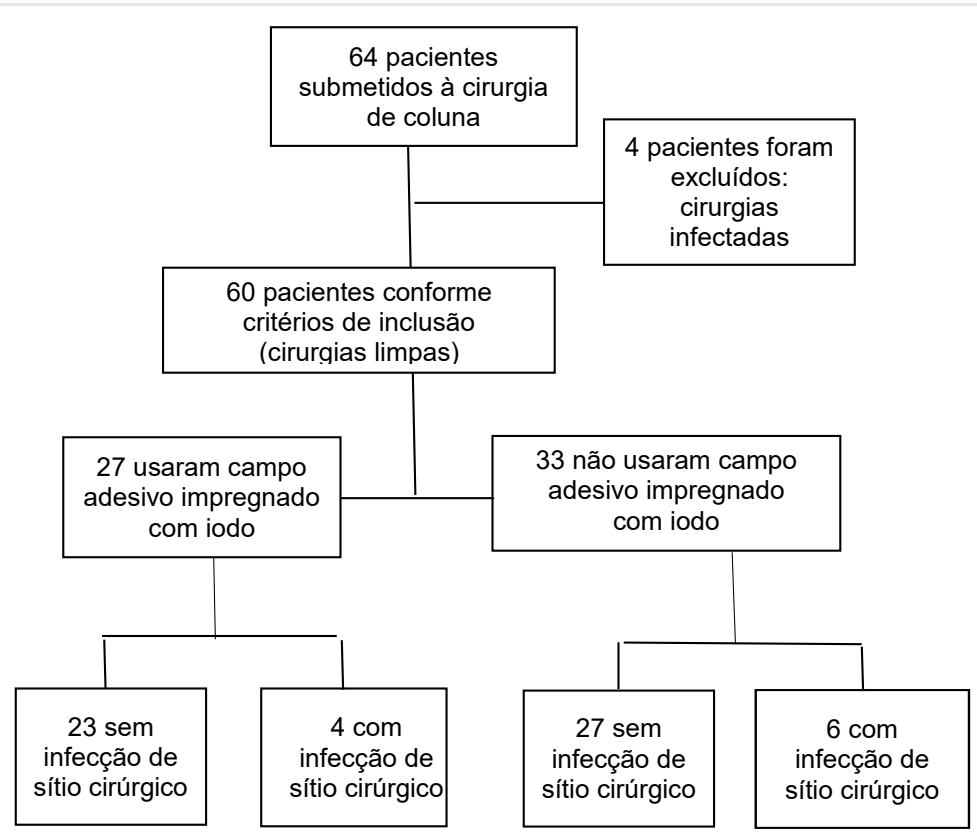

Figura 1. Fluxograma dos pacientes submetidos a cirurgias de coluna em um hospital de ensino no interior do Rio Grande do Sul (2015 a 2019). 


\section{RESULTADOS}

No total, 60 pacientes foram pareados conforme o uso de CAII e o diagnóstico de ISC. Na Tabela 1, identificamos os participantes do estudo e as variáveis basais. Os pacientes que usaram CAII, quando comparados com os que não usaram, foram estatisticamente diferentes em classificação do escore ASA III, tempo de permanência com dreno $>24$ horas,

Tabela 1. Caracterização dos pacientes submetidos à cirurgia de coluna em relação ao uso de campo adesivo impregnado com iodo.

\begin{tabular}{|c|c|c|c|}
\hline & $\begin{array}{l}\text { Uso de CAll } \\
(n=27)\end{array}$ & $\begin{array}{l}\text { Sem uso CAll } \\
\quad(n=33)\end{array}$ & $\mathbf{p}$ \\
\hline Média de Idade (anos/DP) & $59( \pm 17,67)$ & $55( \pm 17,96)$ & $0,346^{*}$ \\
\hline Idade $\leq 60$ anos & $14(51,9)$ & $18(54,5)$ & $0,835^{* *}$ \\
\hline Masculino & $14(51,9)$ & $23(69,7)$ & $0,157^{\star *}$ \\
\hline Urgência cirúrgica & $12(44,4)$ & $12(36,4)$ & $0,971^{* *}$ \\
\hline Atendimento privado de saúde & $22(81,5)$ & $14(42,4)$ & $0,002^{* *}$ \\
\hline Atendimento não privado de saúde & $05(20,8)$ & $19(79,2)$ & $0,002^{* *}$ \\
\hline Internação pré-cirúrgica $\geq 24$ horas & $14(36,8)$ & $24(63,2)$ & $0,216^{* *}$ \\
\hline Internação pré-cirúrgica em UTI & $08(29,6)$ & $09(27,3)$ & $0,840^{* *}$ \\
\hline ASA I & $05(18,5)$ & $01(3,0)$ & $0,257^{* *}$ \\
\hline ASA II & $16(59,3)$ & $26(78,8)$ & $0,212^{* *}$ \\
\hline ASA III & $06(22,2)$ & $06(18,2)$ & $0,050^{* *}$ \\
\hline $\mathrm{IMC}<25 \mathrm{~kg} / \mathrm{m}^{2}$ & $07(25,9)$ & $12(36,4)$ & $0,387^{* *}$ \\
\hline IMC $25-29,9 \mathrm{~kg} / \mathrm{m}^{2}$ & $10(37,0)$ & $15(45,5)$ & $0,511^{* *}$ \\
\hline $\mathrm{IMC}>30 \mathrm{~kg} / \mathrm{m}^{2}$ & $10(37,0)$ & $06(18,2)$ & $0,100^{* *}$ \\
\hline Duração da cirurgia (horas/DP) & $4,8( \pm 1,35)$ & $4,0( \pm 1,38)$ & $0,000^{*}$ \\
\hline Duração cirurgia $>4$ horas & $19(54,3)$ & $16(45,7)$ & $0,087^{\star *}$ \\
\hline Degermação cutânea & $27(100,0)$ & $33(100,0)$ & $0,529^{* *}$ \\
\hline Antissepsia cutânea & $27(100,0)$ & $33(100,0)$ & $0,051^{\star *}$ \\
\hline Uso de profilaxia estendida & $27(100,0)$ & $29(87,9)$ & $0,061^{* *}$ \\
\hline Profilaxia antimicrobiana 1a geração & $24(88,9)$ & $24(72,7)$ & $0,119^{* *}$ \\
\hline Profilaxia antimicrobiana estendida — glicopeptídeos & $03(11,1)$ & $05(15,2)$ & $0,647^{* *}$ \\
\hline Normotermia $\left(\mathrm{T}>35,5^{\circ} \mathrm{C}\right)$ & $13(48,1)$ & $16(48,5)$ & $0,979^{* *}$ \\
\hline Presença de dreno & $27(100)$ & $30(90,9)$ & $0,108^{* *}$ \\
\hline Permanência de dreno $>24$ horas & $06(22,2)$ & $17(51,5)$ & $0,008^{* *}$ \\
\hline Cirurgias "altas" & $05(18,5)$ & $14(42,4)$ & $0,048^{* *}$ \\
\hline Cirurgias "baixas" & $22(81,5)$ & $19(57,6)$ & $0,048^{* *}$ \\
\hline Trauma cirúrgico & $03(11,1)$ & $00(0,0)$ & $0,049^{* *}$ \\
\hline Incidência de ISC & $04(14,8)$ & $06(18,2)$ & $0,728^{* *}$ \\
\hline ISC superficial & $00(0,0)$ & $02(6,06)$ & $0,163^{\star *}$ \\
\hline ISC profundo & $02(7,40)$ & $04(12,12)$ & $0,545^{\star *}$ \\
\hline ISC órgão e espaço & $02(7,40)$ & $00(0,0)$ & $0,112^{* *}$ \\
\hline Internação em UTI pós-operatória (dias/DP) & $3,3( \pm 1,90)$ & $2,7( \pm 1,90)$ & $0,001^{*}$ \\
\hline Internação em UTI pós-operatória & $10(37)$ & $11(33,3)$ & $0,765^{\star *}$ \\
\hline Evolução para óbito & $01(3,7)$ & $00(0,0)$ & $0,529^{* *}$ \\
\hline
\end{tabular}

*Teste $t$ de Student; **teste $\chi^{2}$; CAll: campo adesivo impregnado com iodo; DP: desvio padrão; UTI: unidade de tratamento intensivo; ASA: American Society of Anesthesiologists; IMC: índice de massa corpórea $\left(\mathrm{kg} / \mathrm{m}^{2}\right)$; T: temperatura $\left({ }^{\circ} \mathrm{C}\right)$; ISC: infecção de sítio cirúrgico. 
cirurgia "alta" e "baixa", tempo cirúrgico (horas), trauma cirúrgico e internação pós-cirúrgica em UTI (dias) no pós-operatório imediato, todos com $\mathrm{p} \leq 0,05$. O uso de profilaxia antimicrobiana não apresentou diferença entre o grupo que utilizou campo impregnado, e os pacientes que usaram CAII não apresentaram reações adversas cutâneas associadas.

$\mathrm{Na}$ Tabela 2, as variáveis preditoras para ISC foram: normotermia (risco relativo $-\mathrm{RR}=0,37$;C95\% 0,10-1,31; $\mathrm{p}$
$=0,050)$, tempo cirúrgico (horas) $(\mathrm{RR}=0,22$; IC95\% 0,096,$11 ; \mathrm{p}=0,000$ ), média de tempo de internação (dias) pós-cirúrgica em UTI (RR = 0,32; IC95\% 0,12-5,85; p = 0,003). Não houve diferenças entre a incidência de infecção nos grupos que utilizaram ou não CAII. A frequência geral de ISC foi de $16,7 \%(10 / 60)$, com a taxa de ISC para os pacientes que utilizaram CAII sendo de $40 \%$ e entre os que não utilizaram de $60 \%$ ( $\mathrm{p}=0,728$; IC95\% 0,19-3,11).

Tabela 2. Caracterização dos pacientes submetidos à cirurgia de coluna em relação à presença de infecção.

\begin{tabular}{|c|c|c|c|}
\hline & $\begin{array}{l}\text { Sem ISC } \\
(n=50)\end{array}$ & $\begin{array}{l}\text { Com ISC } \\
(n=10)\end{array}$ & $\mathbf{p}$ \\
\hline Média de Idade (anos/DP) & $54,94( \pm 17,49)$ & $56,80( \pm 20,28)$ & $0,766^{*}$ \\
\hline$\leq 60$ anos & $27(54,0)$ & $05(50,0)$ & $0,817^{\star *}$ \\
\hline Masculino & $32(64,0)$ & $05(50,0)$ & $0,406^{* *}$ \\
\hline Urgência cirúrgica & $07(14,0)$ & $02(20,0)$ & $0,628^{* *}$ \\
\hline Atendimento privado de saúde & $28(56,0)$ & $08(80,0)$ & $0,157^{* *}$ \\
\hline Atendimento não privado de saúde & $22(44,0)$ & $02(20,0)$ & $0,157^{\star *}$ \\
\hline Internação pré-operatória $\geq 24$ Horas & $33(66,0)$ & $05(50,0)$ & $0,338^{* *}$ \\
\hline Internação pré-cirúrgica em UTI & $15(30,0)$ & $02(20,0)$ & $0,522^{* *}$ \\
\hline ASA I & $06(12,0)$ & $00(0,0)$ & $0,248^{* *}$ \\
\hline ASA II & $35(70,0)$ & $07(70,0)$ & $1,000^{* *}$ \\
\hline ASA III & $09(18,0)$ & $03(30,0)$ & $0,386^{* *}$ \\
\hline $\mathrm{IMC}<25 \mathrm{~kg} / \mathrm{m}^{2}$ & $16(32,0)$ & $03(30,0)$ & $0,901^{* *}$ \\
\hline IMC $25-29,9 \mathrm{~kg} / \mathrm{m}^{2}$ & $21(42,0)$ & $04(40,0)$ & $0,907^{* *}$ \\
\hline $\mathrm{IMC}>30 \mathrm{~kg} / \mathrm{m}^{2}$ & $13(26,0)$ & $03(30,0)$ & $0,794^{* *}$ \\
\hline Duração da cirurgia (horas/DP) & $4,2( \pm 1,36)$ & $5,0( \pm 1,48)$ & $0,000^{*}$ \\
\hline Duração cirúrgica $>4$ horas & $29(58,0)$ & $6(60,0)$ & $0,907^{\star *}$ \\
\hline Degermação cutânea & $50(100,0)$ & $10(100,0)$ & $0,019^{* *}$ \\
\hline Antissepsia cutânea & $50(100,0)$ & $10(100,0)$ & $0,047^{* *}$ \\
\hline Uso de profilaxia cirúrgica & $50(100,0)$ & $10(100,0)$ & $0,355^{\star *}$ \\
\hline Profilaxia antimicrobiana 1a geração & $42(84,0)$ & $6(60,0)$ & $0,083^{* *}$ \\
\hline Profilaxia antimicrobiana estendida - glicopeptídeos & $05(10,0)$ & $03(30,0)$ & $0,089 * *$ \\
\hline Uso de CAll & $23(46,0)$ & $04(40,0)$ & $0,728^{* *}$ \\
\hline Normotermia $\left(\mathrm{T}>35,5^{\circ} \mathrm{C}\right)$ & $27(54,0)$ & $02(20,0)$ & $0,050 * *$ \\
\hline Presença de dreno & $47(94,0)$ & $10(100,0)$ & $0,427^{* *}$ \\
\hline Permanência de dreno $>24$ Horas & $26(52,0)$ & $08(80,0)$ & $0,149^{* *}$ \\
\hline Cirurgia “alta” & $18(36,0)$ & $01(10,0)$ & $0,107^{* *}$ \\
\hline Cirurgia "baixa” & $32(64,0)$ & $09(90,0)$ & $0,107^{* *}$ \\
\hline Internação em UTI pós-operatória (dias/DP) & $2,6( \pm 2,03)$ & $04( \pm 1,79)$ & $0,003^{*}$ \\
\hline Internação em UTI pós-operatória & $15(30,0)$ & $06(60,0)$ & $0,069^{* *}$ \\
\hline Evolução para óbito & $00(0,0)$ & $01(10,0)$ & $0,066^{* *}$ \\
\hline
\end{tabular}

*Teste $t$ de Student; **teste $\chi^{2}$; ISC: infecção de sítio cirúrgico; DP: desvio padrão; z UTI: unidade de tratamento intensivo; ASA: American Society of Anesthesiologists; IMC: índice de massa corpórea $\left(\mathrm{kg} / \mathrm{m}^{2}\right)$; T: temperatura $\left({ }^{\circ} \mathrm{C}\right)$; CAll: campo adesivo impregnado com iodo. 
As infecções ocorreram em nove (90\%) cirurgias "baixas". Seis $(60 \%)$ pacientes necessitaram de UTI no pós-operatório, com média de quatro $( \pm 2)$ dias. A média de internação hospitalar foi de $31( \pm 2)$ dias.

Observamos dois $(6,1 \%)$ casos de ISC superficial, com incidência nos pacientes que não usaram CAII ( $\mathrm{p}=0,193$; IC95\% 0,09-0,28) - um caso confirmado por Serratia spp. e outro que manteve cultura negativa. Essas infecções aconteceram em pacientes submetidos a cirurgias "baixas". As infecções ocorreram na média em cinco $( \pm 2)$ dias de pós-operatório, com média de 15 ( \pm 9) dias de internação hospitalar.

A ISC profunda aconteceu em dois $(7,4 \%)$ pacientes que usaram CAII e em quatro $(12,2)$ que não usaram ( $\mathrm{p}=0,545$; IC95\% 0,00-0,29). Destes, foram isolados dois com Enterobacter spp. e um com Klebsiella spp.; nos demais pacientes não foram encontrados microrganismos nas culturas. As infecções ocorreram em média em 33 ( \pm 44) dias, cinco $(83,3 \%)$ delas até o décimo sexto dia. Dos seis pacientes que tiveram ISC profundo, quatro $(66,7 \%)$ tiveram internação em UTI no pós-operatório, com média de permanência de três $( \pm 2)$ dias e média de internação hospitalar de $36( \pm 18)$ dias. Essas infecções (5/6) aconteceram em pacientes submetidos a cirurgias "baixas".

Em dois (7,4\%) pacientes que usaram CAII ocorreu a ISC órgão e espaço, apesar de a diferença não ter sido significativa ( $p=0,112$; IC95\% 0,13-0,34). Em ambas as cirurgias houve ruptura de dura-máter e não houve crescimento de microrganismos na cultura. As infecções ocorreram em aproximadamente nove $( \pm 1)$ dias em pacientes com pós-operatório em UTI, com média de permanência de cinco $( \pm 1)$ dias e média de internação hospitalar de 28 ( \pm 14$)$ dias.

Em relação ao IRIC dos procedimentos analisados, $47(78,3 \%)$ cirurgias apresentaram IRIC igual a 1 , incluindo-se nessa categoria sete (70,0\%) infecções do estudo ( $\mathrm{p}=0,483$; IC95\% 0,00-0,28). Na análise geral, o controle glicêmico pré e pós-operatório foi obtido em $50 \%$ desses pacientes, e a abordagem cirúrgica posterior ocorreu em 33 (55,0\%) deles, com nove $(27,3 \%)$ casos de ISC.

Foi identificado um $(1,7)$ desfecho de óbito no $25^{\circ}$ dia pós-operatório e com ISC profunda em paciente feminina, maior de 60 anos, com IRIC $=2$, ASA III, obesa e com comorbidades (diabetes e cardiopatia).

As variáveis com $\mathrm{p}<0,20$ foram associadas com as variáveis $\operatorname{com} \mathrm{p}<0,05$ para análise multivariada de fatores de risco para ISC, que são: sistema de saúde para internação ( $\mathrm{p}=0,157)$, profilaxia antimicrobiana estendida com cefalosporina $(\mathrm{p}=$ $0,083)$, profilaxia antimicrobiana estendida com glicopeptídeos $(\mathrm{p}=0,089)$, tempo de dreno cirúrgico $>24$ horas $(\mathrm{p}=0,149)$, cirurgia "alta" e "baixa” ( $\mathrm{p}=0,107)$, internação pós-operatória em UTI ( $p=0,069)$ e óbito $(p=0,069)$.

A Tabela 3 destaca, na regressão logística, a normotermia $\mathrm{p}<0,05$ ( $\mathrm{RR}=0,27$; IC95\%, 0,01-0,40), que manteve associação com a ISC. Demais variáveis testadas mantiveram $\mathrm{p}>0,05$, não sendo comprovado nível de significância.

O custo dos procedimentos foi de $\mathrm{R} \$ 3.031 .388,7$ (US\$ 571.960,13), e R\$ 1.135.125,7 (US\$ 214.174,66) foi relacionado aos dez pacientes que tiveram infecção. Entre os 50 pacientes que não desenvolveram infecção, o custo foi de R\$ 1.896.263,0 (US\$ 357.785,47).

Em média, os custos foram de R \$1.936,99/ dia (US\$ 365,47/ dia) nos pacientes que não apresentaram infecção e de R\$ 3.615,05/dia (US\$ 682,08/ dia) nos que desenvolveram infecção. A principal diferença foram os custos relacionados aos tratamentos de complicações infecciosas, com incremento de $83,63 \%$ / dia de internação, pois os pacientes que tiveram eventos infecciosos apresentaram maior média de permanência hospitalar, de $31,4( \pm 17,4)$ dias, em relação à dos que não desenvolveram essa situação, de 19,6 ( \pm 14,2) dias, ou seja, 11,8 $( \pm 3,2)$ dias a mais.

O custo dos pacientes que não usaram CAII para a cura foi superior ao dos que usaram, embora a diferença não tenha sido significativa ( $\mathrm{p}=0,728$; IC95\% 0,00-0,33). Independentemente do desfecho de infecção ou não, o estudo

Tabela 3. Regressão linear múltipla de variáveis preditoras da infecção de sítio cirúrgico em pacientes submetidos a cirurgias de coluna.

\begin{tabular}{|c|c|c|c|c|}
\hline & $\begin{array}{r}\text { Sem ISC } \\
(n=50)\end{array}$ & $\begin{array}{l}\text { Com ISC } \\
(n=10)\end{array}$ & p & RR IC95\% \\
\hline Normotermia $\left(\mathrm{T}>35,5^{\circ} \mathrm{C}\right)$ & $27(54,0)$ & $02(20,0)$ & 0,043 & $0,269(0,006-0,402)$ \\
\hline Cirurgia "baixa" & $32(64,0)$ & $09(90,0)$ & 0,277 & $0,153(0,104-0,353)$ \\
\hline Internação em UTI pós-operatória & $15(30,0)$ & $06(60,0)$ & 0,242 & $0,166(0,092-0,356)$ \\
\hline Profilaxia antimicrobiana - cefalosporina 1a geração & $42(84,0)$ & $06(60,0)$ & 0,229 & $0,196(0,501-0,123)$ \\
\hline
\end{tabular}

ISC: infecção de sítio cirúrgico; RR: risco relativo; IC95\%: intervalo de confiança de 95\%. 
mostrou que a média de permanência hospitalar dos pacientes que não usaram CAII foi de 26 ( \pm 17 ) dias, e a dos que usaram o CAII foi de $16( \pm 10)$ dias.

\section{DISCUSSÃO}

A utilização de CAII para a prevenção de infecção em cirurgias de coluna vertebral não apresentou significância estatística neste estudo. Sugere-se, pela análise das variáveis clínicas na comparação entre os pacientes que utilizaram CAII versus os que não utilizaram, que isso possa ter ocorrido em razão da não uniformidade do agrupamento, pois tal fato não foi controlado. Os pacientes que utilizaram CAII foram considerados mais graves, ou seja, apresentaram maior frequência no escore ASA III, maior tempo cirúrgico, trauma de dura-máter (pré e transoperatório) e internação pós-cirúrgica em UTI.

Os fatores de proteção contra ISC foram: normotermia, menor tempo cirúrgico e menor tempo de internação pós-operatória em UTI. No entanto, na regressão linear, permaneceu apenas a normotermia como variável independente, que é até mesmo recomendação de diversas diretrizes nacionais e internacionais ${ }^{11-14}$. No entanto, uma metanálise sugere não associação com ISC, mas os autores propõem novas pesquisas ${ }^{15}$. Um estudo demonstrou que a incidência de hipotermia nos pacientes cirúrgicos é alta, aumentando no decorrer do procedimento e presente na sala de recuperação pós-anestésica. Os autores destacam a necessidade de educação permanente da equipe em relação à aplicação de medidas preventivas para evitar esse agravo ${ }^{16}$.

$\mathrm{Na}$ análise das demais variáveis, embora não significativas estatisticamente, a ISC superficial não foi identificada nos pacientes que usaram CAII. Tal fato possivelmente tenha ocorrido pela propriedade de diminuir a microbiota transitória, mesmo das camadas mais profundas da pele (1.000 micrometros), em virtude da tecnologia de maior concentração e permanência do antisséptico ${ }^{5}$. Um estudo concluiu que o CAII, em cirurgias ortopédicas, reduz significativamente as bactérias que colonizam a pele da incisão, diminuindo a probabilidade teórica de $\mathrm{ISC}^{17}$. Outra pesquisa demonstrou a diminuição da ISC superficial em cirurgias cardíacas ${ }^{4}$.

A ISC profunda não apresentou diferença quando se usou ou não CAII. As meningites apresentavam como fator de risco o trauma de dura-máter. Como esperado, o CAII não tem ação perante as complicações traumáticas profundas ${ }^{4}$, à qual a tecnologia não se propõe. Este estudo encontrou frequências de ISC categorizadas pelo IRIC maior do que em outra pesquisa analisada ${ }^{18}$.

Salienta-se que os procedimentos cirúrgicos de maior proximidade com a coluna lombossacra apresentam possibilidade de microbioma tecidual maior, especialmente com maior recolonização dos tecidos no trans e pós-operatório. Um dos objetivos da antissepsia de pele é diminuir, principalmente, bactérias do gênero Staphylococcus spp., principal agente de ISC em coluna ${ }^{19}$.

A ISC em coluna vertebral varia entre 1 e $13 \%$, e a cirurgia de abordagem posterior apresenta maior incidência de infecção do que a realizada pela via anterior ${ }^{19,20}$. Outro fator relacionado é a programação cirúrgica, com a intervenção em vários níveis intervertebrais². Tais achados geram um questionamento sobre as variáveis confundidoras entre ISC superficial e profunda, o que pode gerar resultados conflitantes sobre os benefícios diretos e indiretos do CAII ${ }^{6}$.

Esta análise não demonstrou que o sobrepeso acrescentou risco de ISC, embora ele estivesse presente em $70 \%$ das ISC (superficiais e profundas). Em contrapartida, essa relação foi encontrada em outro estudo ${ }^{2}$. Existem evidências de que, para cada $1 \mathrm{~mm}$ de espessura de gordura do subcutâneo, são acrescentados $6 \%$ no risco de ISC, com aumento de até quatro vezes para pacientes com espessura de gordura acima de $50 \mathrm{~mm}$ na região lombar ${ }^{21}$.

O controle glicêmico pós-operatório estava adequado em metade dos pacientes analisados e, por ser uma variável de fácil mensuração e de baixo custo, deve ser prioritário e encorajado pela equipe cirúrgica.

A mortalidade geral foi baixa, sendo comparável com a de outro estudo. A idade, o sexo feminino e as comorbidades influenciam no risco de complicações pós-operatórias e no índice de mortalidade ${ }^{22}$.

Os pacientes que não apresentaram ISC tiveram permanência hospitalar diminuída e, consequentemente, menores custos com atendimento. Determinar a relação custo-benefício do uso do CAII, embora este não tenha significativo em reduzir ISC, demonstrou outra grande vantagem, que foi a redução dos custos totais do atendimento hospitalar ${ }^{4}$. Salientamos que os pacientes que utilizaram CAII tiveram $\operatorname{dez}( \pm 4,9)$ dias a menos de permanência hospitalar. Houve maior prescrição de antimicrobianos, reintervenções cirúrgicas, necessidade de anestesia e de exames complementares. A redução dos custos de hospitalização é um importante 
parâmetro para avaliar a eficácia do procedimento cirúrgico e de tratamentos coadjuvantes.

\section{LIMITAÇÕES DO ESTUDO}

Como limitação deste estudo, destacamos o fato de ter sido uma investigação retrospectiva, com poucos sujeitos, sem controle dos que utilizaram ou não CAII e com aplicação nos pacientes com maiores riscos. Tal fato pode ter acarretado o resultado de não haver diferenças nas taxas de ISC. A positividade da cultura de microrganismos foi baixa, o que também dificulta a análise dos agentes infecciosos mais prevalentes. Variáveis que são fatores de risco conhecidos não foram coletadas por não estarem registradas nos prontuários físico e informatizado.

\section{CONCLUSÕES}

Os resultados do presente estudo sugerem que o uso de CAII não foi associado à redução estatisticamente significativa de ISC. Apenas a normotermia foi fator de proteção para ISC na regressão linear. Os custos com complicações relacionadas ao sítio cirúrgico ou à distância aumentaram o valor da hospitalização, e o grupo dos pacientes em uso de CAII obteve redução dos gastos totais do atendimento. Esses dados podem ser úteis para o planejamento cirúrgico, o aconselhamento dos pacientes e para traçar esforços a fim de melhorar a segurança e a relação custo-benefício das cirurgias de coluna. Sugerimos que estudos prospectivos, controlados e multicêntricos sejam realizados, de modo a evidenciar resultados positivos.

\section{REFERÊNCIAS}

1. Saleh A, Thirukumaran C, Mesfin A, Molinari RW. Complications and readmission after lumbar spine surgery in elderly patients: an analysis of 2,320 patients. Spine J. 2017;17(8):1106-12. https:// doi.org/10.1016/j.spinee.2017.03.019

2. Fei $Q$, Li J, Lin J, Li D, Wang B, Meng H, et al. Risk factors for surgical site infection after spinal surgery: a meta-analysis. World Neurosurg. 2016;95:507-15. https://doi.org/10.1016/j. wneu.2015.05.059

3. Yao R, Zhou H, Choma TJ, Kwon BK, Street J. Surgical site infection in spine surgery: who is at risk? Global Spine J. 2018;8(4):5S30S. https://doi.org/10.1177/2192568218799056

4. Bejko J, Tarzia V, Carrozzini M, Gallo M, Bortolussi G, Comisso $M$, et al. Comparison of efficacy and cost of iodine impregnated drape vs. standard drape in cardiac surgery: study in 5100 patients. J Cardiovasc Transl Res. 2015;8(7):431-7. https://doi. org/10.1007/s12265-015-9653-1

5. Casey AL, Karpanen TJ, Nightingale P, Conway BR, Elliott TS. Antimicrobial activity and skin permeation of iodine present in an iodine-impregnated surgical incise drape. J Antimicrob Chemother. 2015;70(8):2255-60. https://doi.org/10.1093/jac/ dkv100

6. Dumville JC, McFarlane E, Edwards P, Lipp A, Holmes A. Preoperative skin antiseptics for preventing surgical wound infections after clean surgery. Cochrane Database Syst Rev. 2013;(3):CD003949. https://doi.org/10.1002/14651858.cd003949. pub3

7. Brasil. Agência Nacional de Vigilância Sanitária (ANVISA). Critérios diagnósticos de infecções relacionadas à assistência à saúde. Brasília: ANVISA; 2017 [acessado em 10 out. 2020]. Disponível em: https://www.gov.br/anvisa/pt-br/centraisdeconteudo/ publicacoes/servicosdesaude/publicacoes/caderno-2-criteriosdiagnosticos-de-infeccao-relacionada-a-assistencia-a-saude. $\mathrm{pdf} /$ view

8. Brasil. Agência Nacional de Vigilância Sanitária (ANVISA). Nota Técnica GVIMS / GGTES n 03 / 2019. Critérios diagnósticos das infecções relacionadas à assistência à saúde. Brasília: ANVISA; 2019 [acessado em 10 out. 2020]. Disponível em: https://www.gov. br/anvisa/pt-br/centraisdeconteudo/publicacoes/servicosdesaude/ notas-tecnicas/nota-tecnica-no-2-2019-gvims-ggtes-anvisa.pdf/view

9. American Society of Anesthesiologists (ASA). ASA House of Delegates/ Executive Committee. Physical Status Classification System. American Society of Anesthesiologists; 2019 [acessado em 10 out. 2020]. Disponivel em: https://www.asahq.org/standards-and-guidelines/ asa-physical-status-classification-system.

10. National Nosocomial Infections Surveillance System. National Nosocomial Infections Surveillance (NNIS) system report: data summary from January 1992 to June 1996. Am J Infect Control. 2004;32(8):470-85. https://doi.org/10.1016/s0196655304005425

11. Brasil. Agência Nacional de Vigilância Sanitária (ANVISA). Medidas de prevenção de infecção relacionada à assistência à saúde. Brasília: ANVISA; 2017. [acessado em 10 out. 2020]. Disponível em: https://www.gov.br/anvisa/pt-br/centraisdeconteudo/publicacoes/ servicosdesaude/publicacoes/caderno-4-medidas-de-prevencaode-infeccao-relacionada-a-assistencia-a-saude.pdf/view

12. Leaper DJ, Edmiston CE. World Health Organization: global guidelines for the prevention of surgical site infection. J Hosp Infect. 2017;95(2):135-6. https://doi.org/10.1016/j.jhin.2016.12.016

13. Garner BH, Anderson DJ. Surgical site infections: an update. Infect Dis Clin North Am. 2016;30(4):909-29. https://doi.org/10.1016/j. idc.2016.07.010 
14. Centers for Disease Control and Prevention (CD). National Healthcare Safety Network National Healthcare Safety Network (NHSN) Patient Safety Component Manual. Estados Unidos: CDC; 2020 [acessado em 10 out. 2020]. Disponivel em: https://www.cdc.gov/nhsn/PDFs/ pscManual/pcsManual_current.pdf

15. Bu N, Zhao E, Gao Y, Zhao S, Bo W, Kong Z, et al. Association between perioperative hypothermia and surgical site infection: a meta-analysis. Medicine (Baltimore). 2019;98(6):e14392. https://doi.org/10.1097/ MD.0000000000014392

16. Ribeiro E, Navarro NT, Armede VCB, Rodrigues HS, do Valle JP, Duran ECM. Frequência de hipotermia não intencional no perioperatório de cirurgias eletivas. Rev. SOBECC. 2016;21(2):68-74. https://doi. org/10.5327/Z1414-442520160002000

17. Rezapoor M, Tan TL, Maltenfort MG, Parvizi J. Incise draping reduces the rate of contamination of the surgical site during hip surgery: a prospective, randomized trial. J Arthroplasty. 2018;33(6):1891-5. https://doi.org/10.1016/j.arth.2018.01.013

18. Culver DH, Horan TC, Gaynes RP, Martone WJ, Jarvis WR, Emori TG, et al. Surgical wound infection rates by wound class, operative procedure, and patient risk index. National Nosocomial Infections Surveillance System. Am J Med. 1991;91(3 Supl. 2):S152-7. https:// doi.org/10.1016/0002-9343(91)90361-z

19. Zhou J, Wang R, Huo X, Xiong W, Kang L, Xue Y. Incidence of surgical site infection after spine surgery: a systematic review and meta-analysis. Spine (Phila Pa 1976). 2020;45(3):208-16. https://doi.org/10.1097/ BRS. 000000000003218

20. Pull ter Gunne AF, Cohen DB. Incidence, prevalence, and analysis of risk factors for surgical site infection following adult spinal surgery. Spine (Phila Pa 1976). 2009;34(13):1422-8. https://doi.org/10.1097/ BRS.0b013e3181a03013

21. Lee JJ, Odeh KI, Holcombe SA, Patel RD, Wang SC, Goulet JA, et al. Fat thickness as a risk factor for infection in lumbar spine surgery. Orthopedics. 2016;39(6):e1 124-e8. https://doi.org/10.3928/01477447-20160819-05

22. Schoenfeld AJ, Ochoa LM, Bader JO, Belmont PJ. Risk factors for immediate postoperative complications and mortality following spine surgery: a study of 3,475 patients from the national surgical quality improvement program. J Bone Joint Surg. 2011;93(17):1577-82. https://doi.org/10.2106/JBJS.J.01048 